\title{
A case of relapsing Salmonella osteomyelitis in a thalassaemia trait patient
}

\author{
F. Rayan · C. Mukundan · D. D. Shukla
}

Received: 16 February 2008/Accepted: 14 October 2008/Published online: 22 November 2008

(C) Springer-Verlag 2008

\begin{abstract}
We report a case of chronic relapsing osteomyelitis caused by Salmonella Stanley in a $\beta$-thalassaemia trait patient who is otherwise normal. The importance of obtaining definitive bacteriological diagnosis and timely intervention to treat bone infection effectively is emphasised here.
\end{abstract}

Keywords Salmonella . Osteomyelitis .

Haemoglobinopathies · Thalassaemia

\section{Introduction}

There have been various case reports of Salmonella osteomyelitis in sickle cell anaemia $[1,2]$. We present the first case of Salmonella Stanley causing a relapsing bone infection at the same site 12 years apart in a patient with $\beta$-thalassaemia trait. There is an increased incidence of foodborne salmonellosis in England [3]. Systemic infections may therefore become of increasing importance for clinicians. Currently, no clinical studies or antibiotic and surgical guidelines exist to guide treatment of Salmonella osteomyelitis.

\section{Case report}

A 36 year old male was referred to the accident and emergency department with a suspicion of septic arthritis

F. Rayan · C. Mukundan - D. D. Shukla

Kettering General Hospital, Kettering, UK

F. Rayan ( $\square)$

No. 15 Moorhouseway, Kettering NN15 7LX, UK

e-mail: rayanmarakkar@gmail.com of the right ankle. He complained of swelling and pain of three days duration with no history of trauma, an inability to bear weight, and the patient had an unremitting fever for two days. He had a history of infection of the right ankle 18 years back, which was treated with antibiotics only, and he had also been diagnosed with $\beta$-thalassaemia trait. On examination he was hemodynamically stable, the right ankle was swollen and erythematous, and ankle movements were restricted and painful. A complete blood profile was done, and it suggested a raised WCC of $9.3 \times 10^{9} / \mathrm{L}$ and a CRP of $228 \mathrm{mg} / \mathrm{L}$.

AP and lateral radiographs of the right ankle (Figs. 1 and 2) showed mixed areas of radiodensity and radiolucency in the medial malleolus, with no periosteal reaction. Aspirate of the ankle joint did not yield any organisms, and blood cultures were negative.

He was started on intravenous antibiotics for three days, but the patient continued to suffer further spikes in temperature, increasing swelling and worsening of the pain.

His inflammatory markers were on the rise. The patient was taken to theatre for exploration and decompression of the right ankle. Twenty millilitres of pus were drained through an anteromedial incision. Following the creation of a cortical window, purulent material was drained from the medullary cavity and specimens of osseous material were sent for culture and sensitivity testing. A curette was used to evacuate any loculation of pus in the medullary canal. Thorough debridement and irrigation of the area was performed using $3 \mathrm{~L}$ of normal saline. Betadine-soaked gauze was then loosely packed into the intramedullary canal to allow postoperative drainage.

The patient developed pneumonic consolidation and was hence transferred to the intensive care unit for $24 \mathrm{~h}$; he subsequently stabilised while on antibiotic therapy for the chest infection. Further debridement was carried out in 


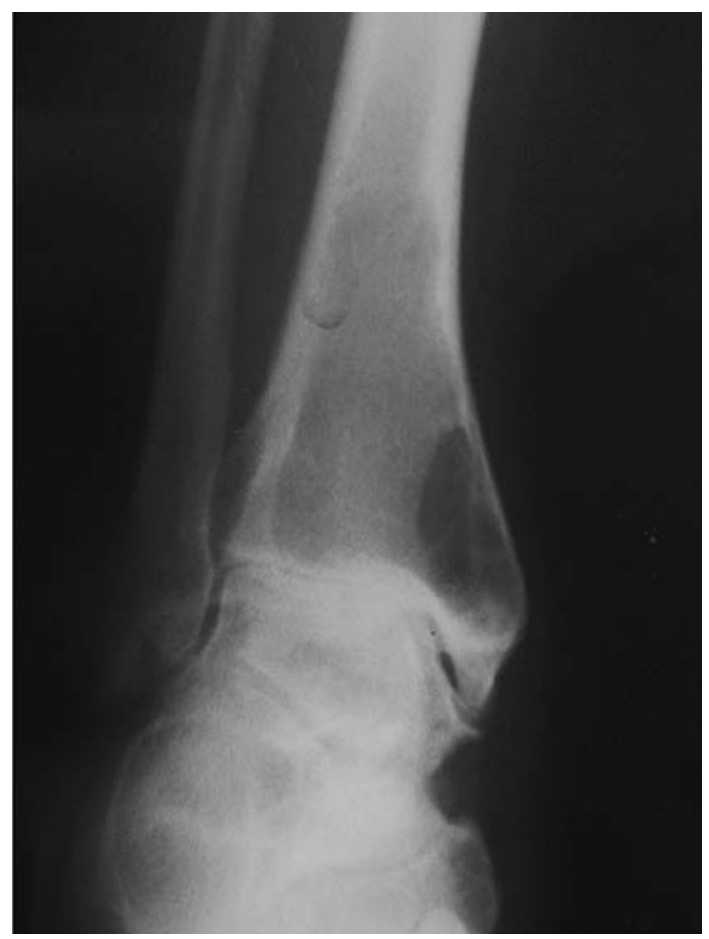

Fig. 1 AP radiograph of the ankle showing the characteristic lesion in the distal tibia

$48 \mathrm{~h}$, and a final washout and secondary closure was done after another $48 \mathrm{~h}$. Microbiology culture and sensitivity results showed Salmonella Stanley that was sensitive to gentamicin and ciprofloxacin. He was started on intravenous gentamicin and ciprofloxacin for two weeks, followed by oral ciprofloxacin for six weeks. For two weeks the wound was clean and dry. The inflammatory markers WCC and CRP slowly fell to near normal. He was discharged and followed up in the community periodically due to the chronic nature of this disease.

\section{Discussion}

It was Hodges and Holt in 1951 who reported an increasing frequency of Salmonella osteomyelitis in sickle cell disease [1]. Salmonella is a Gram-negative nonsporing bacillus belonging to the family of Enterobacteriaceae. Most cases are from contaminated food or water, with the small bowel being the habitat for the organism. Most often it results in self-limiting gastroenteritis requiring no antibiotics. Various other extraintestinal Salmonella infections, like meningitis and osteomyelitis, have been reported $[4,5]$.

Salmonella osteomyelitis is very rare $(0.45 \%$ of all osteomyelitis), and osteitis is reported in only $0.8 \%$ of cases of typhoid fever [6]. The common way in which this infection spreads is haematogenously; the most frequent sites involved are the diaphyses of long bones and

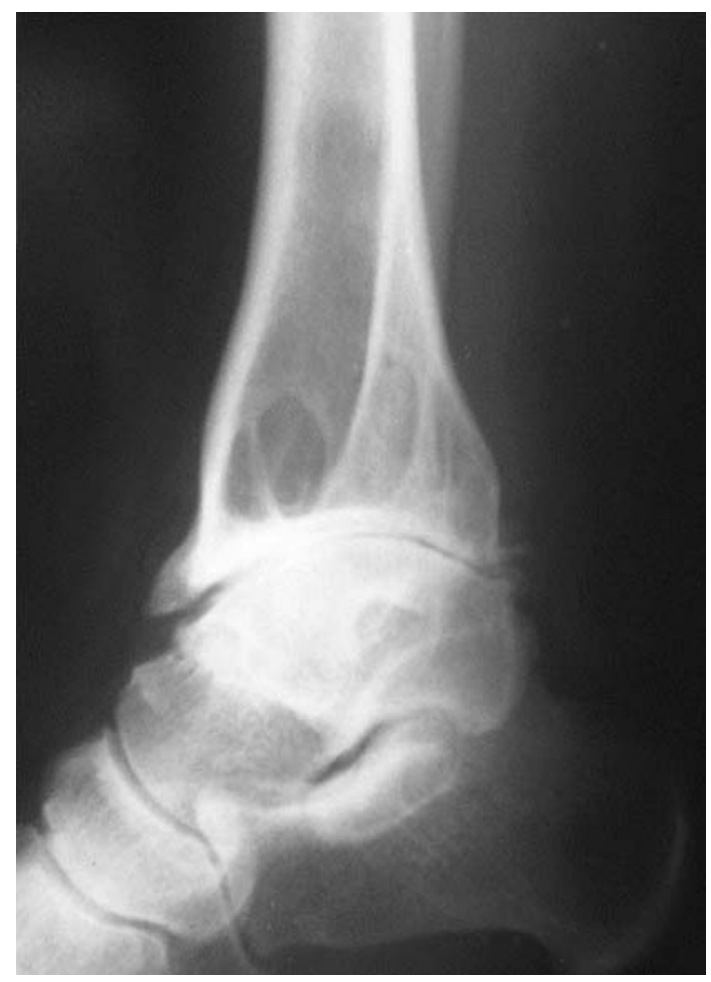

Fig. 2 Lateral radiograph of the ankle showing the characteristic lesion in the distal tibia

vertebrae $[7,8]$. This can be attributed to increased bone marrow activity and increased blood degradation products, which favours the growth of Salmonella [1,9]. The association of Salmonella osteomyelitis with sickle cell anaemia and other haemoglobinopathies is well recognised [8]. A preceding history of infection is not usual [10]. Stool cultures may or may not be positive [10]. Serological tests for Salmonella are of limited value under these circumstances [10].

Various reports suggest that non-Typhi serotypes are frequently the causative agent $[8,11-13]$.

Salmonella Stanley subtypes have been isolated from a certain brand of peanuts in three countries, namely Australia, Canada and the UK [14]. These subtypes are fully sensitive to antibiotics and have a distinct pulsed field gel electrophoresis profile. Salmonellosis is a global problem due to the international movement of foods and its high incidence in exporting countries. In September 2001, Australia isolated Salmonella Stanley from imported peanuts in an outbreak investigation, which resulted in wider investigations in Canada, England, Wales and Scotland [14].

There is no consensus regarding the treatment of acute and chronic osteomyelitis, as no randomised or case-control studies have been performed. The antibiotic treatment for osteomyelitis should ideally be initiated after obtaining sufficient samples from bone for microbiological assay. 
Ampicillin, chloramphenicol, third-generation cephalosporins and quinolones have been used alone or in combination [15]. Hyperbaric oxygen therapy has also been used as an adjunct [15]. Ciprofloxacin has been recommended for the treatment of chronic bone infections, as it can achieve adequate levels in the bone. There are few reports of relapsing osteomyelitis in the literature [16, 17]. Lang et al. [17] described a unique case of two episodes of Salmonella paratyphi $\mathrm{C}$ osteomyelitis at different sites in the lower limbs 17 years apart. There is no consensus in the literature on ways to prevent chronic or relapsing Salmonella osteomyelitis. Initial investigations are full blood count, inflammatory markers (ESR, CRP), radiographs and bone scan, followed by MRI. These modalities help to identify bone infections [15].

The patient was given IV gentamycin and ciprofloxacin for two weeks followed by oral ciprofloxacin for a further six weeks. The duration of the antibiotics was based on clinical features and inflammatory markers. Due to the chronic nature of the infection the patient is still being followed up at our institution.

The patient provided informed consent to the publication of this clinical case.

Conflict of interest statement The authors declare that they have no conflict of interest related to the publication of this manuscript.

\section{References}

1. Engh CA, Hughes JL, Abrams RC, Bowerman JW (1971) Osteomyelitis in the patient with sickle-cell disease. J Bone Joint Surg Am 53(1):1-15

2. Sadat-Ali M (1998) The status of acute osteomyelitis in sickle cell disease. A 15-year review. Int Surg 83(1):84-87

3. Nathwani D, Morris AJ, Laing RB, Smith CC, Reid TM (1991) Salmonella virchow: abscess former amongst the contemporary invasive Salmonellae? Scand J Infect Dis 23(4):467-471

4. Ispahani P, Slack RC (2000) Enteric fever and other extraintestinal salmonellosis in University Hospital, Nottingham, UK, between 1980 and 1997. Eur J Clin Microbiol Infect Dis 19(9):679-687

5. Cohen JI, Bartlett JA, Corey GR (1987) Extra-intestinal manifestations of Salmonella infections. Medicine (Baltimore) 66(5):349-388

6. Ingram R, Redding P (1988) Salmonella virchow osteomyelitis. A case report. J Bone Joint Surg Br 70(3):440-442

7. Pallares R, Costa J, Villabona C, Capell S, Garau J (1982) Salmonella typhi osteomyelitis. Med Clin (Barc) 79(7):339

8. Caspi I, Ezra E, Lin E, Horoszowski H, Rubinstein E (1986) Salmonella osteomyelitis after internal fixation of fracture. Orthop Rev 15(3):174-176

9. Rao SP, Miller S, Solomon N (1986) Acute bone and joint manifestations of sickle cell disease in children. N Y State J Med 86(5):254-260

10. Deysine M, Isenberg HD, Steiner G (1983) Chronic haematogenous osteomyelitis; studies on an experimental model. Int Orthop 7(2):69-78

11. Monsivais JJ, Scully TJ, Dixon BL (1988) Chronic osteomyelitis of the hand caused by Salmonella typhimurium. A case report. Clin Orthop Relat Res 226:231-234

12. Schnabel T, Koster W, von Wersebe O (1990) Salmonella osteomyelitis involving multiple bones in chronic myeloid leukemia. Rontgenblatter 43(8):359-361

13. Tomczak RL, Melonakos A, Mabry MG, Wrege S (1988) Acute hematogenous Salmonella osteomyelitis in a nonsickle cell infant. J Am Podiatr Med Assoc 78(10):535-538

14. Kirk MD, Little CL, Lem M, Fyfe M, Genobile D, Tan A, Threlfall J, Paccagnella A, Lightfoot D, Lyi H, McIntyre L, Ward L, Brown DJ, Surnam S, Fisher IS (2004) An outbreak due to peanuts in their shell caused by Salmonella enterica serotypes Stanley and Newport-sharing molecular information to solve international outbreaks. Epidemiol Infect 132(4):571-577

15. Banky JP, Ostergaard L, Spelman D (2002) Chronic relapsing Salmonella osteomyelitis in an immunocompetent patient: case report and literature review. J Infect 44(1):44-47

16. Montejo M, Aguirrebengoa K, Garcia-Alonso JA, Urberuaga M, Ruiz-Irastorza G, de la Prieta R, Montes M (1994) Chronic osteomyelitis caused by Salmonella fyris. Clin Infect Dis 19$1: 221$

17. Lang R, Maayan MC, Lidor C, Savin H, Kolman S, Lishner M (1992) Salmonella paratyphi C osteomyelitis: report of two separate episodes 17 years apart. Scand J Infect Dis 24(6):793796 